\title{
Large-angle scattered light measurements for quantum-noise filter cavity design studies
}

\author{
Fabian Magaña-Sandoval, ${ }^{1, *}$ Rana X. Adhikari, ${ }^{2}$ Valera Frolov, ${ }^{3}$ Jan Harms, ${ }^{2}$ Jacqueline Lee, ${ }^{1}$ \\ Shannon Sankar, ${ }^{4,5}$ Peter R. Saulson, ${ }^{6}$ and Joshua R. Smith ${ }^{1}$ \\ ${ }^{1}$ Department of Physics, California State University Fullerton, Fullerton, California 92831, USA \\ ${ }^{2}$ LIGO Laboratory-California Institute of Technology, Pasadena, California 91125, USA \\ ${ }^{3}$ LIGO_Livingston Observatory, Livingston, Louisiana 70754, USA \\ ${ }^{4}$ LIGO Laboratory-Massachusetts Institute of Technology, Cambridge, Massachusetts 02139, USA \\ ${ }^{5}$ Currently with Department of Physics, University of Colorado, Boulder, Colorado 80309-0440, USA \\ ${ }^{6}$ Department of Physics, Syracuse University, Syracuse, New York 13244, USA \\ ${ }^{*}$ Corresponding author: fabianmagana@csu.fullerton.edu
}

Received April 25, 2012; accepted June 25, 2012; posted June 27, 2012 (Doc. ID 167231); published July 27, 2012

\begin{abstract}
Optical loss from scattered light could limit the performance of quantum-noise filter cavities being considered for an upgrade to the Advanced Laser Interferometer Gravitational Wave Observatory (LIGO) gravitational-wave detectors. This paper describes imaging scatterometer measurements of the large-angle scattered light from two high-quality sample optics, a high reflector and a beamsplitter. These optics are each superpolished fused silica substrates with silica:tantala dielectric coatings. They represent the current state-of-the art optical technology for use in filter cavities. We present angle-resolved scatter values and integrate these to estimate the total scatter over the measured angles. We find that the total integrated light scattered into larger angles can be as small as $4 \mathrm{ppm}$. (c) 2012 Optical Society of America
\end{abstract}

OCIS codes: $\quad 290.1483,120.5820,110.0110,230.1360,120.3180$.

\section{INTRODUCTION}

A second generation of gravitational-wave (GW) detectors is scheduled to start operation within the next 5 to 10 years [1-3]. Quantum noise of the light is expected to limit the sensitivity of these detectors over a wide range of frequencies. An R\&D plan has been defined that addresses the possibility of upgrading the Advanced Laser Interferometer Gravitational Wave Observatory (LIGO) detectors after some years of operation [4]. The upgrades will further increase the significance of quantum noise at frequencies above $10 \mathrm{~Hz}$. Advanced techniques to mitigate quantum noise, such as the implementation of squeezed light, are currently being tested in large-scale interferometers [5].

To optimize the benefit from squeezed light and also to minimize the effect of optical radiation-pressure noise, it will be necessary to optically filter squeezed-light fields [6,7] (e.g., with triangular cavities). The foremost challenge when using squeezed light is to reduce the optical losses inside the interferometer. The benefit from filter cavities depends strongly on the cavity round-trip loss that can be realized. Filter-loss requirements depend on the cavity length and the main interferometer configuration, but for a $50 \mathrm{~m}$ long filter cavity, the round-trip loss should not exceed a value of about $20 \mathrm{ppm}$. Table 1 summarizes previous loss measurements on cavities of various sizes. Round-trip loss in the kilometer-scale arm cavities of the second-generation GW detectors not including the input transmission is expected to be of order $100 \mathrm{ppm}$. These values indicate that short cavities typically have lower loss than long cavities. One of the questions is why this is the case.
The main loss mechanism in these cavities is scattering from the optics. The quality of currently available optics does not seem to be sufficient to allow us to build weak scattering (low-loss) filter cavities. Scattering is caused by point defects on the mirror surface or inside the mirror coating $[13,14]$, by residual surface roughness after substrate polishing and coating [15-17], and by larger-scale figure errors [18]. Ultimately, the loss contribution of each scattering process needs to be known and linked to some deficiency of the coating and substrate fabrication process.

Figure errors and surface roughness are currently the dominant cause of scattering in long cavities. The reason is that the beam size on the optics increases with cavity size and therefore the beam is sensitive to larger-scale mirror errors. Errors at larger scales are typically worse than small-scale errors. Decreasing the beam size, losses get smaller until one eventually comes into the regime where scattering is dominated by point defects. The density of point defects is often very high and a beam is scattered from many defects simultaneously. In contrast, the very small loss values in Table 1 can only be realized by steering a very small beam into a region of the mirror surface that has no significant defects. The beam size in a $50 \mathrm{~m}$ cavity would be about $8 \mathrm{~mm}$, which is larger than the typical distance between defects on current optics. If this is truly the case, then the only solution to the scattering problem in filter cavities is to improve current coating and substrate technology. As a first step to understand quality limits in the fabrication of optics, we measured scattering from two high-quality optics as a function of scattering angle, which allows us to draw further conclusions about the scattering process involved. The optics made of superpolished fused silica 
Table 1. Summary of Cavity-Loss Measurements ${ }^{a}$

\begin{tabular}{lccl}
\hline Length $[\mathrm{m}]$ & Beam radii $[\mathrm{mm}]$ & $\mathrm{Lpm}^{a}[\mathrm{ppm}]$ & Year \\
\hline 10 & $1.9 / 2.1$ & 60 & $84[\underline{8}]$ \\
0.004 & $0.084 / 0.085$ & 1.1 & $92[\underline{\underline{9}}]$ \\
0.202 & $0.37 / 0.41$ & 1.5 & $96[\underline{\underline{10}}]$ \\
0.202 & $0.37 / 0.41$ & 1.6 & $98[\underline{11}]$ \\
20 & $2.2 / 3.8$ & 30 & $99[\underline{\underline{12}}]$ \\
\hline
\end{tabular}

${ }^{a} \mathrm{Lpm}=$ Loss per mirror

substrates with silica:tantala dielectric coatings represent the current state-of-the art optical technology that could be used in filter cavities.

\section{EXPERIMENT}

\section{A. Samples}

The optics we measured were both 2 in diameter fused silica substrates, with superpolished surfaces, and ion-sputtered dielectric coatings (see, e.g., Chap. 2 in [19]) composed of alternating layers of silica and tantala.

The beamsplitter (BS) we measured is shown in Fig. 1. It was coated by Advanced Thin Films of Boulder, Colorado, for use at 1064 and $532 \mathrm{~nm}$ wavelengths and with a $45^{\circ}$ nominal angle of incidence. We measured an $18.4 \%$ reflectivity and $81.4 \%$ transmission for horizontally polarized $1064 \mathrm{~nm}$ light incident at $45^{\circ}$. The barrel of this optic is unpolished and it scatters brightly even for a small beam incident on the center of the optical surface. The bright scatter from the barrel is also mirrored by the front and back surfaces of the optic.

The highly reflecting mirror (HRM) is shown in Fig. 2. It was manufactured by Gooch and Housego (S/N 13589) for operation at $1064 \mathrm{~nm}$ wavelength. The transmission of the HRM was measured using a $1064 \mathrm{~nm}$ laser and calibrated power meter to be roughly $20 \mathrm{ppm}$ from $0-35^{\circ}$ for $p$-polarization. The barrel of this optic is polished, and scattered much less than that of the BS.

\section{B. Experimental Setup}

Figure 3 shows the layout of the imaging scatterometer that was used to characterize the forward-scattering of laser light from our optics. Its basic operation is as follows. A laser beam illuminates the optical sample at a fixed incidence angle. Images of the light scattered from the sample's surface(s) are recorded over a discrete set of scattering angles, $\theta_{s}$, defined as the angle between the camera's imaging direction and the normal to the sample surface [20]. The scattering angle is adjusted using a motorized rotation stage. The angles, with respect to the incident beam, that are accessible by this setup range from the smallest angle that allows the imaging optics to see past the fiber launch $\left(5^{\circ}\right)$ to the angle where
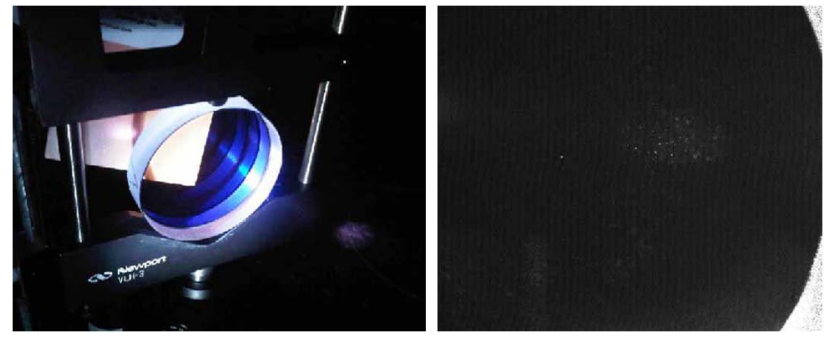

Fig. 1. (Color online) The BS shown in room light (left) and as viewed by our setup with $1064 \mathrm{~nm}$ laser illumination (right).
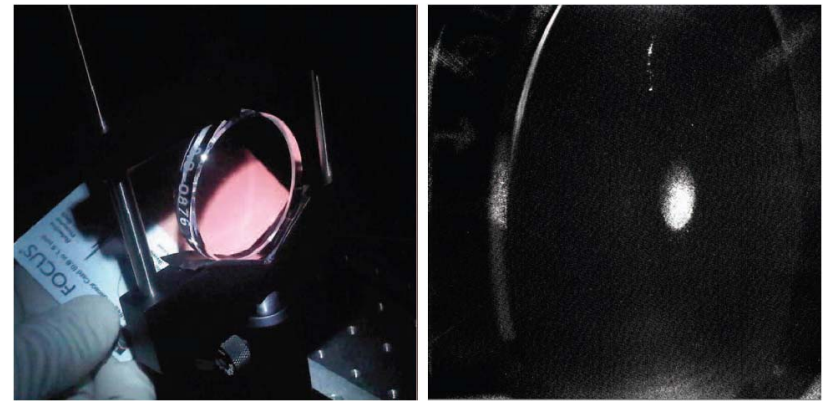

Fig. 2. (Color online) The HRM shown in room light (left) and as viewed by our setup with $1064 \mathrm{~nm}$ laser illumination (right).

the optical surface is parallel to the charge-coupled device (CCD) camera $\left(90^{\circ}\right.$ for normal incidence). A consequence of this is that when the angle of incidence on the sample is near normal, only large-angle scattering can be measured.

The incident laser power is provided by a linearly polarized, $1064 \mathrm{~nm}$ nominally $500 \mathrm{~mW}$ laser (model: CrystaLaser CL 1064-500-SO). The laser beam is guided to the setup by an optical fiber that includes a 90:10 BS. The 10\% fiber is directed to a photodiode for power monitoring, while the $90 \%$ fiber is directed to a series of optics mounted on the rotation stage. When the laser exits the narrow fiber aperture, it is strongly diverging, so we first collimate it to an $8.5 \mathrm{~mm}$ beam diameter using a reflective collimator (Thorlabs RC08FC). Then a linear polarizer is used to further improve the laser polarization and ensure that it is horizontal (parallel to the tabletop). Finally, an adjustable iris is set to approximately $6 \mathrm{~mm}$ diameter to clip the beam edges and reduce the amount of light falling on the sample holder, which has a much higher scatter coefficient than the samples, and can contaminate the images. The beam incident on the sample is thus horizontally polarized with approximately $8 \mathrm{~mm}$ diameter waist, truncated to a diameter of $6 \mathrm{~mm}$, and has about $150 \mathrm{~mW}$ of power.

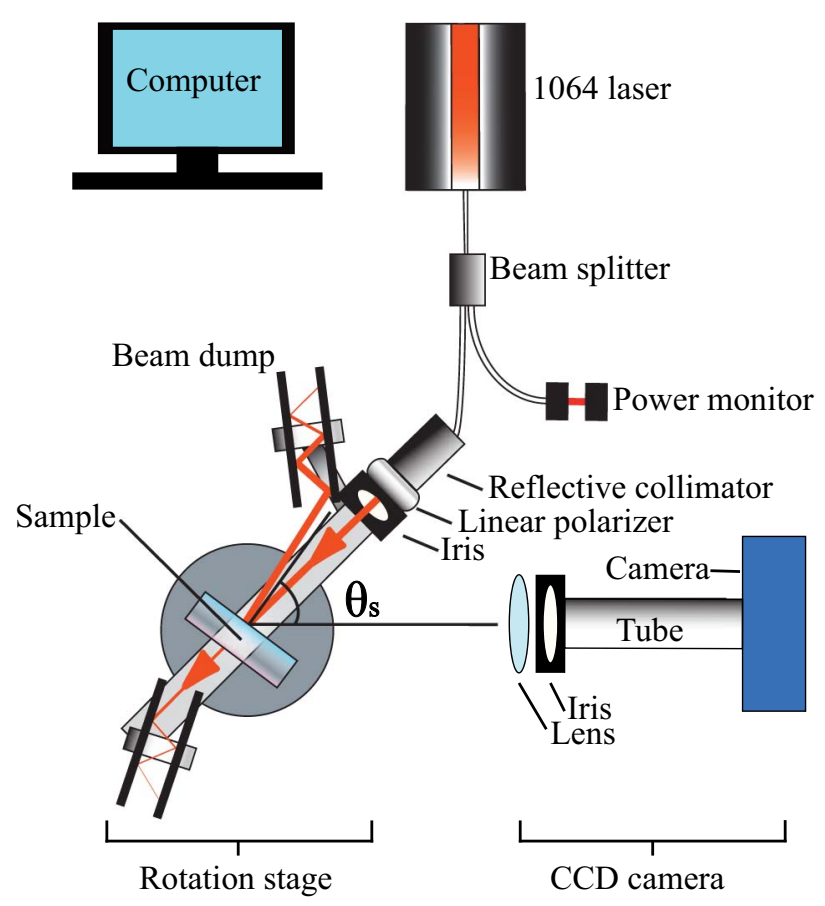

Fig. 3. (Color online) The layout of the imaging scatterometer. 
The optical sample, the fiber-coupled laser output, and two black-glass beam traps are mounted on a motorized rotation stage. This ensures that as the stage is rotated, the angle of incidence remains fixed and the reflected and transmitted beams are dumped. The center of the front surface of the optic is positioned directly above the rotation axis of the stage so that when the stage is rotated the optic does not translate in the images.

The imaging system views the optical surface at an angle with respect to the input beam axis. A single 2 in diameter $f=$ $100 \mathrm{~mm}$ biconvex lens and adjustable iris with diameter set to $16 \mathrm{~mm}$ are located about $50 \mathrm{~cm}$ from the sample and form an image of the optical surface on a 1 megapixel astronomical CCD) camera (Apogee Alta U6). Between the iris and the camera, an aluminum tube with an RG850 optical high pass filter at its entrance is used to reduce room lights and unwanted stray laser light from reaching the camera.

Measurements are performed by taking a camera exposure at a given scattering angle, while also recording the input laser power, then rotating the stage a small amount (such as $1^{\circ}$ ) and repeating. The camera exposure times are adjusted to provide a good scattered light signal-to-noise ratio, while not saturating parts of the image close to the laser beam spot on the sample surface(s). For angles near to the intense specular reflection from the $\mathrm{BS}$, the camera saturates even at its shortest exposure time $(0.02 \mathrm{~s})$. These angles have been removed from our analysis.

Prior to measurement, the front and back surfaces of each sample were drag-wiped with an optical tissue and methanol to remove dust and impurities. The incidence angle was set to $45^{\circ}$ for the BS and $3^{\circ}$, the smallest angle that allowed dumping the back-reflected beam cleanly in the black-glass trap, for the HRM. The setup is housed in a soft-wall clean room that uses overpressure and laminar flow to reduce airborne particles.

\section{Calibration}

For our analysis, we need to convert the image counts measured by the CCD camera into a calibrated measure of scatter. To do this, we measure the scattered light from a diffusing sample twice, once with the CCD camera and once with a calibrated power meter. We then compare their readings. This uses the setup as described above, but with (i) a diffusing target (Spectralon Diffusion Material, $1^{\prime \prime} \times 0.012^{\prime \prime}$ disk, SM-00875200 ) as the sample $[21,22]$, (ii) an additional power meter that can be inserted in front of the imaging optics to measure scattered light power, and (iii) a neutral-density filter with a measured 1/273 transmission factor at $1064 \mathrm{~nm}$ inserted in front of the CCD camera to prevent saturation. Images of the diffusing sample in room light and with incident laser light and viewed via the CCD camera are shown in Fig. 4.

For these calibrations, the power meter was located $21.6 \mathrm{~cm}$ from the center of the sample surface, and at the height of the laser beam (which is parallel to the tabletop). With it we recorded the scattered power over a set of scattering angles separated by $10^{\circ}$ steps. From these measurements, we calculated the standard bidirectional reflectance distribution function [20],

$$
\mathrm{BRDF}=\frac{P_{s}}{P_{i} \Omega \cos \theta_{s}},
$$

where $P_{i}$ is the incident laser power, and $P_{s}$ is the scattered light power detected by the power meter, which subtends a
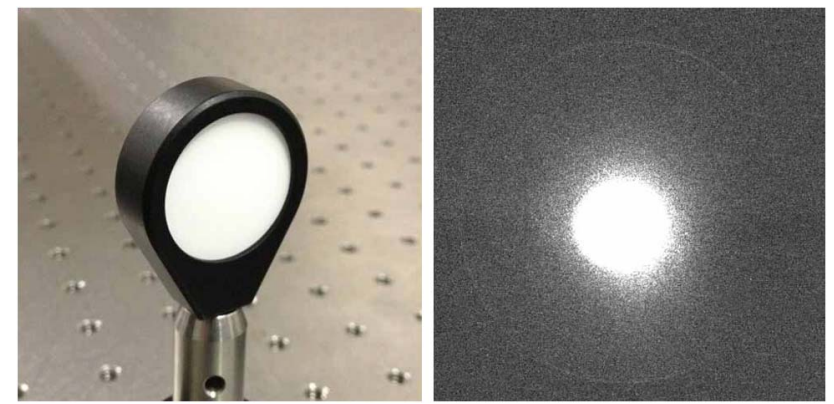

Fig. 4. (Color online) The diffusing sample shown in room light (left) and as viewed by our setup with $1064 \mathrm{~nm}$ laser illumination (right).

solid angle $\Omega$, and is oriented at polar angle $\theta_{s}$ with respect to the normal to the optical surface (in the plane of the laser beam).

Then we used the CCD camera to take images for the same scattering angles. For each image, a region of interest (ROI) corresponding to the area of the sample surface with significant light power incident on it is chosen. The counts over the entire ROI are summed and normalized by the camera exposure time $T_{\exp }$ and incident laser power on the sample to give

$$
\mathrm{ARB}_{\mathrm{CCD}}=\frac{\sum_{k} V_{k}}{T_{\exp } P_{i}},
$$

where $V_{k}$ is the value of the $k$ th pixel in the ROI.

We use the fact that the BRDF is intrinsic to the sample to calculate a calibration function,

$$
F=\frac{\mathrm{BRDF} \cos \left(\theta_{s}\right)}{\mathrm{ARB}_{\mathrm{CCD}}}
$$

that relates the CCD counts to the BRDF measured by the power meter.

Figure 5 shows the results of the calibration used for this paper. We measured a mean BRDF of $0.27 \mathrm{STR}^{-1}$ for our diffuse scattering sample for near normal incidence. This value is consistent with BRDF measured for similar targets [21,23]. The normalized counts measured by the CCD behave as we would expect, falling off as the cosine of the scattering angle. Following Eq. (3), we calculate a calibration constant of $F=3.20 \times 10^{-14} \mathrm{~W}$ sec Counts $^{-1} \mathrm{STR}^{-1}$. This value is used in the following analysis.

\section{Data Analysis}

As described above, the data products measured for each sample are megapixel CCD images taken over a range of discrete scattering angles. The procedure for analyzing the images and producing BRDF curves is as follows. First a dark image (an image with the same exposure time, but with the laser turned off) is subtracted from each image, removing the camera noise and any hot pixels. Then we select for each image an ROI, a rectangular area that captures the vast majority of the light scattered by the surface of the optic, while including as little as possible of the scattering from the optic barrel or the optic mount. An ROI encompassing scatter from both front and back surfaces was used for the BS, because significant amounts of scattered light was visible from each surface (the fact that the scatter from the back surface is diminished by two passes through the $81.4 \%$ reflectivity front 

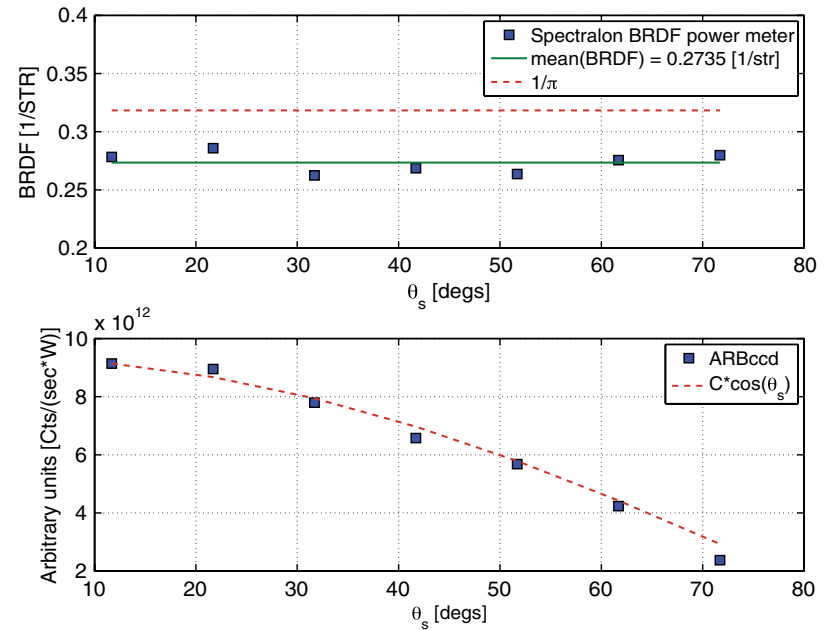

Fig. 5. (Color online) The top graph shows BRDF measured by the power meter and a comparison with the $1 / \pi$ value expected for an ideal Lambertian diffuser (uniform hemispherical scattering; see Chapter 11 in [19]). The bottom graph shows the counts recorded by the CCD and normalized by the exposure time and incident power. This is compared to the $\cos \left(\theta_{s}\right)$ scattered power dependency expected for an ideal Lambertian diffuser.

surface was not taken into account). The scatter from the HRM was dominated by scatter from the front surface so only that was included in the ROI. Then we sum the pixel values in the ROI of the subtracted image to calculate $\mathrm{ARB}_{\mathrm{CCD}}$, following Eq. (2), and finally calibrate the values into BRDF using the calibration constant given above, and the relation

$$
\mathrm{BRDF}=\mathrm{ARB}_{\mathrm{CCD}} F .
$$

Figure 6 shows a set of four example subtracted images for the BS. The scatter from the front and back surfaces can be seen to separate as the sample is rotated through scattering angles from -20 to $59^{\circ}$. The ROI is indicated with a rectangular line. Note that the unpolished barrel of the BS scatters

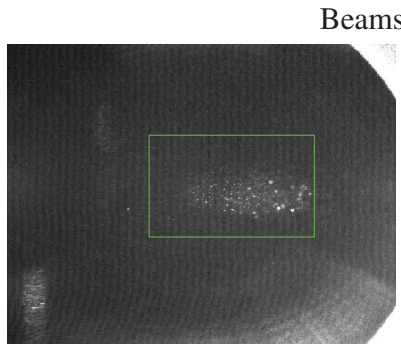

Theta_s $=-20$ degrees

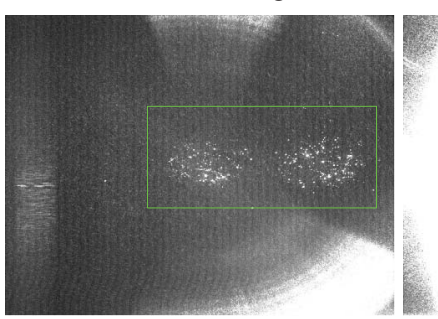

Theta_s $=21$ degrees

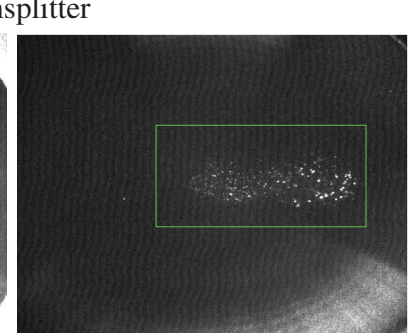

Theta_s $=0$ degrees

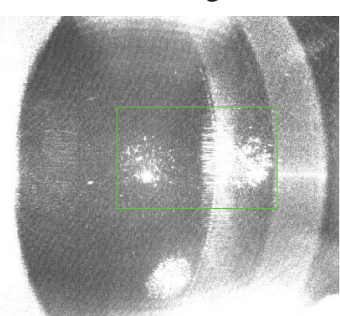

Theta_s $=59$ degrees
Fig. 6. (Color online) The BS viewed at scattering angles of $-20^{\circ}$ (upper left), $0^{\circ}$ (upper right), $21^{\circ}$ (lower left), and $59^{\circ}$ (lower right). The rectangles are the regions of interest that are used to measure the scattered light.

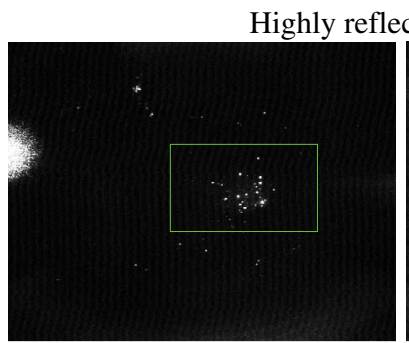

Theta_s $=13$ degrees

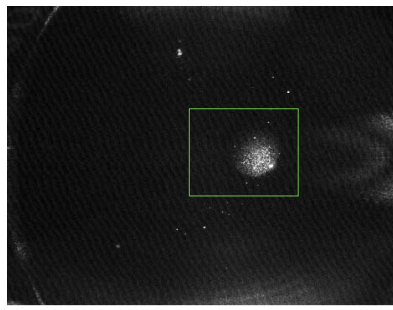

Theta_s $=44$ degrees

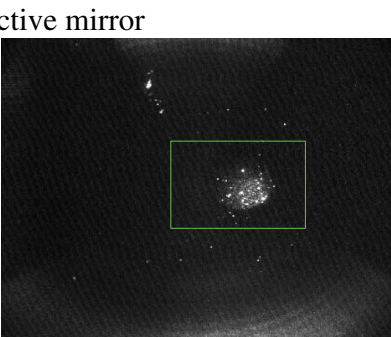

Theta_s $=27$ degrees

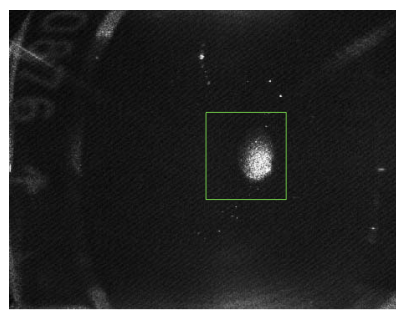

Theta_s $=58$ degrees
Fig. 7. (Color online) The HRM viewed at scattering angles of $13^{\circ}$ (upper left), $27^{\circ}$ (upper right), $44^{\circ}$ (lower left), and $58^{\circ}$ (lower right). The rectangles are the regions of interest that are used to measure the scattered light.

brightly, and that its reflections off the mirror surfaces pollute the ROI for large viewing angles. Figure 7 shows four separate scattering images over a range of angles from the HRM. Here there is much less background scatter with respect to the BS because the polished barrel of the HRM scatters far less. Also, the regions of interest encompass only the front surfaces. As the scattering angle gets larger, the scattering character changes from a constellation of point scatterers to a diffuse glow.

Figures 6 and 7 show two distinct types of scattering. Where the beam is most intense on the sample surface, a strong central scattering "glow" is seen. This has a diffuse speckle pattern that is characteristic of scatter caused by surface roughness. In this region, and even well outside of this region where the laser power is significantly less, a sparse constellation of bright points can be seen. We find that some of these points can be removed or relocated by drag-wiping, indicating that they originate from dust and other impurities on the surface. The images shown correspond to the cleanest data set over several drag wipes, performed in situ in our clean room.

\section{RESULTS}

Figure $\underline{8}$ shows the BRDF results for the BS $\left(45^{\circ}\right.$ angle of incidence) versus scattering angle. This data can be separated into a few distinct regions. In the -40 to $40^{\circ}$ region, the images were similar to the first three panels shown in Fig. 6. Scatter from the front and back surfaces was imaged cleanly without influence from unwanted stray light. Here we see BRDF with values between $1 \times 10^{-6}$ and $1 \times 10^{-5} \mathrm{STR}^{-1}$. In the range from 40 to $58^{\circ}$, the CCD camera was saturated by light from the BS's specular reflection. This data has been removed. The data from 60 to $80^{\circ}$ includes scatter from the BS surfaces, but this is polluted by an increasingly large component of unwanted scattered light from the unpolished barrel of the optic and the optical mount. This BRDF in this region is not to be trusted. 


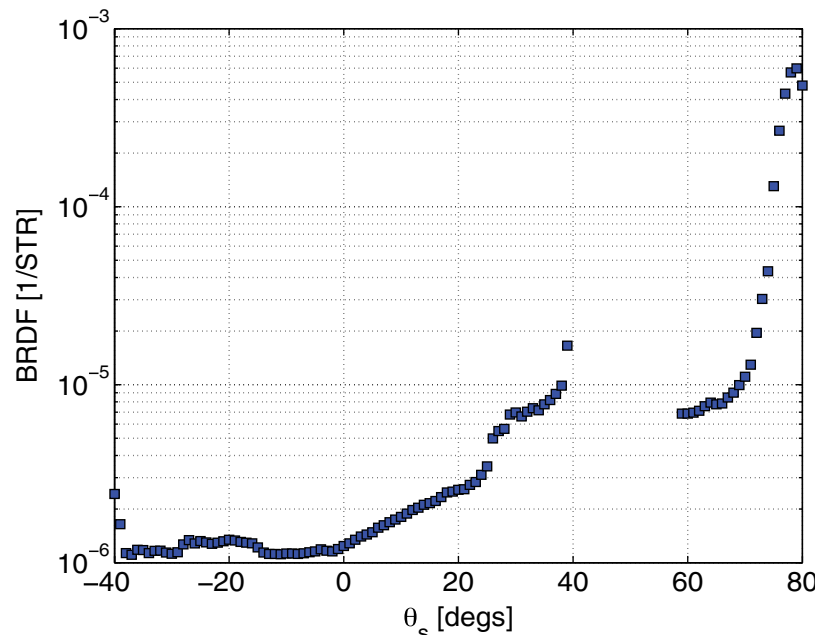

Fig. 8. (Color online) BRDF versus scattering angle for the BS sample. Data points have been removed for angles where the CCD camera saturated due to the strong near-specular reflection at $45^{\circ}$. The BRDF from 60 to $80^{\circ}$ is increasingly overestimated due to spatial overlap with scatter from the unpolished barrel; see lower right image in Fig. $\underline{6}$.

Figure 9 shows the measured BRDF for the HRM. This data was significantly cleaner than for the BS due to less unwanted scattered light polluting the images. The BRDF for this mirror is quite low, below $10^{-6}$ for scattering angles greater than $30^{\circ}$. The BRDF for the HRM was checked using a similar scatterometer, which utilized a single photodetector and chopper wheel, in another lab. For normal incidence we measured $\mathrm{BRDF}=6 \times 10^{-7} \mathrm{STR}^{-1}$ at $\theta_{s}=45^{\circ}$, which is close to the value for that angle in Fig. 9 .

These BRDF values can also be used to estimate the total scattering associated with these optics. Total integrated scatter (TIS) [20] can be estimated by integrating a measurement of BRDF times $\cos \theta_{s}$ over the full solid angle of scatter (a hemisphere for back-scatter), to get the hemispherical reflectance [24],

$$
R_{H}=\frac{P_{s}}{P_{i}}=R \mathrm{TIS}=\int_{0}^{2 \pi} \int_{0}^{\pi / 2} \mathrm{BRDF} \cos \theta_{s} \sin \theta_{s} \mathrm{~d} \theta_{s} \mathrm{~d} \phi_{s},
$$

where $R$ is the reflectivity of the optic, and $\phi_{s}$ are the azimuthal scattering angles. (For a perfect Lambertian diffuser,

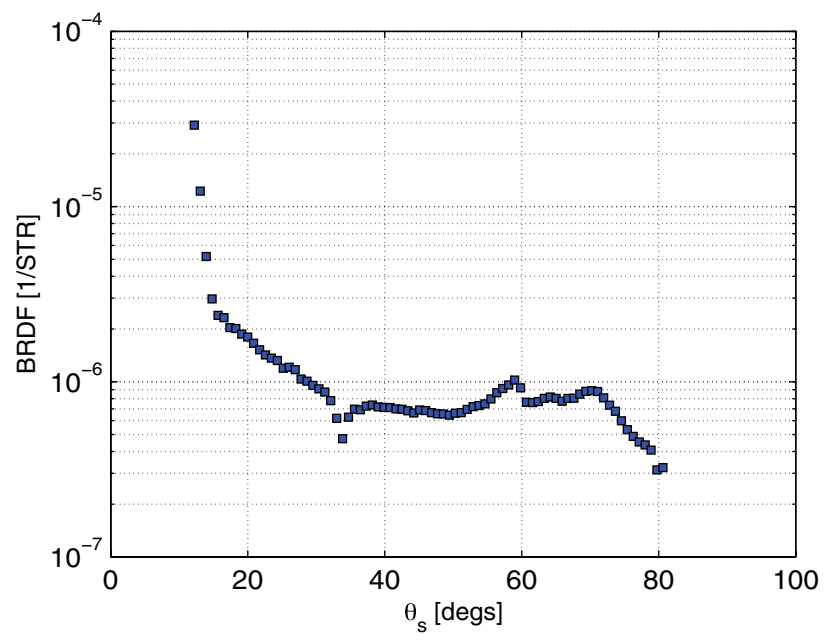

Fig. 9. (Color online) BRDF versus scattering angle for the HRM.
Table 2. TIS Calculated from BRDF Measurements in Figs. $\underline{8}$ and $\underline{9}^{a}$

\begin{tabular}{lcc}
\hline Sample & Angle range [degrees] & R TIS [ppm] \\
\hline BS & {$[-40,0]$} & 1.62 \\
BS & {$[0,37]$} & 5.71 \\
BS & {$[60,80]$} & 57.0 \\
HRM & {$[9,85]$} & 3.79 \\
\hline
\end{tabular}

${ }^{a}$ The $60-80^{\circ}$ range for the BS is polluted by scatter from the optic barrel.

BRDF is constant and $R$ and TIS are unity. Evaluating Eq. (5) with this information leads to $\mathrm{BRDF}=1 / \pi$ for all forwardscattering angles, the value referred to above [19].)

We assume independence of the BRDF function on azimuthal scattering angles, since we use smoothly polished optics that should scatter isotropically at a given polar angle. This allows integration of the scatterometer data over only the polar angle of scatter. We approximate the solid angle integral as a sum of the scatter in individual rings centered on the polar angles of measured scatter $\theta_{s}$,

$$
\begin{aligned}
R \operatorname{TIS}\left(\theta_{s}\right) & =\Omega_{\text {ring }}\left(\theta_{1}, \theta_{2}\right) \operatorname{BRDF}\left(\theta_{s}\right) \cos \theta_{s} \\
& =2 \pi\left(\cos \theta_{1}-\cos \theta_{2}\right) \operatorname{BRDF}\left(\theta_{s}\right) \cos \theta_{s},
\end{aligned}
$$

where $\Omega_{\text {ring }}=2 \pi\left(\cos \theta_{2}-\cos \theta_{1}\right)$ is the solid angle of the ring subtended by polar angles between $\theta_{1}=\theta_{s}-d \theta / 2$ and $\theta_{2}=\theta_{s}+d \theta / 2$, where $d \theta$ is the angular resolution (step) of the scatterometer measurement.

Table 2 shows the TIS values calculated for the BS and HRM using the data presented in Figs. $\underline{8}$ and $\underline{9}$ and the equations above.

\section{DISCUSSION}

The BRDF measurement of a high-reflective optic showed that the total integrated light scattered into larger angles can be as small as $4 \mathrm{ppm}$ provided that the optic is cleaned and the measurement is carried out in a near particle-free environment. Somewhat larger BRDF values were measured for a BS, but as discussed in the text, it is also more challenging to obtain unbiased results for BRDF measurements on optics with nonnegligible transmission and unpolished surfaces.

Large-angle scattering of superpolished optics is generally associated with point defects in mirror coatings. The TIS values shown in Table 2 need to be added to estimates of smallangle surface-roughness scattering that is usually obtained from measured surface-roughness profiles. These profiles are unknown for the two optics measured for this paper, but extrapolating estimates from measured surface-roughness spectra as, for example, presented in [25], one obtains additional scattering of $1 \mathrm{ppm}$ and less. It should be noted, though, that the same article points out that a discrepancy has been observed in the past between measured BRDF and estimates obtained from surface-roughness spectra. Nevertheless, based on the values at hand, it seems possible that filter cavities with round-trip scatter loss less than 20 ppm can be constructed with the available optics.

The main goal in the near future will be to form a consistent picture between BRDF measurements, scattering estimates from surface-roughness measurements, and actual loss observed in optical cavities, for example, through ring-down 
measurements. Further measurements and simulations are necessary to explore the small-angle regime (below $10^{\circ}$ ) and improve our scatter-loss predictions. Even though filter cavities will have lengths of $50 \mathrm{~m}$ and longer, loss measurements on smaller cavities can help to improve our understanding of scattering provided that cavity-loss measurements are combined with scattering measurements on individual optics that form the cavity.

\section{ACKNOWLEDGMENTS}

This work is supported by the Research Corporation for Science Advancement Cottrell College Science Award \#19839 and by National Science Foundation Awards PHY0970147, PHY-0854812, and PHY-0555406. We thank our colleagues in the LIGO Scientific Collaboration for fruitful discussions about this research and for review of this manuscript.

\section{REFERENCES}

1. J. R. Smith for the LIGO Scientific Collaboration, "The path to the enhanced and advanced LIGO gravitational-wave detectors," Class.l Quantum Grav. 26, 114013 (2009).

2. The VIRGO Collaboration, "Advanced virgo baseline design," VIRGO Collaboration, VIR 027A 09 (2009).

3. K. Kuroda (on behalf of the LCGT Collaboration), "Status of LCGT," Class. Quantum Grav. 27, 084004 (2010).

4. The LIGO Scientific Collaboration, "Instrument science white paper," LIGO DCC p. T1100309-v5 (2011). https.dcc.ligo.org/ cgi-bin/DocDB/ShowDocument?docid=62186

5. The LIGO Scientific Collaboration, "A gravitational wave observatory operating beyond the quantum shot-noise limit," Nature Physics 7, 962 (2011).

6. H. J. Kimble, Y. Levin, A. B. Matsko, K. S. Thorne, and S. P. Vyatchanin, "Conversion of conventional gravitational-wave interferometers into quantum nondemolition interferometers by modifying their input and/or output optics," Phys. Rev. D 65, 022002 (2001)

7. J. Harms, Y. Chen, S. Chelkowski, A. Franzen, H. Vahlbruch, K. Danzmann, and R. Schnabel, "Squeezed-input, optical-spring, signal-recycled gravitational-wave detectors," Phys. Rev. D 68, 042001 (2003).

8. D. Z. Anderson, J. C. Frisch, and C. S. Masser, "Mirror reflectometer based on optical cavity decay time," Appl. Opt. 23, 1238-1245 (1984).

9. G. Rempe, R. J. Thompson, H. J. Kimble, and R. Lalezari, "Measurement of ultralow losses in an optical interferometer," Opt. Lett. 17, 363-365 (1992)
10. A. Ueda, N. Uehara, K. Uchisawa, K. Ueda, H. Sekiguchi, T Mitake, K. Nakamura, N. Kitajima, and I. Kataoka, "Ultra-high quality cavity with $1.5 \mathrm{ppm}$ loss at $1064 \mathrm{~nm}$," Opt. Rev. 3, 369-372 (1996).

11. A. Ueda, H. Yoneda, K. Ueda, K. Waseda, and M. O-Hashi, "Two-dimensional measurement of optical parameters of superhigh-quality mirrors," Laser Physics 8, 697-702 (1998).

12. S. Sato, S. Miyoki, M. Ohashi, M. Fujimoto, T. Yamazaki, M. Fukushima, A. Ueda, K. Ueda, K. Watanabe, K. Nakamura, K Etoh, N. Kitajima, K. Ito, and I. Kataoka, "Loss factors of mirrors for a gravitational wave antenna," Appl. Opt. 38, 2880-2885 (1999).

13. The VIRGO Collaboration, "The VIRGO large mirrors: a challenge for low loss coatings," Class. Quantum Grav. 21, S935-S945 (2004).

14. H. Yamamoto, "Effects of small size anomalies in a FP cavity," LIGO DCC T1000154-v5 (2010). https.dcc-llo.ligo.org/cgi-bin DocDB/ShowDocument?docid $=10316$

15. C. J. Walsh, A. J. Leistner, and B. F. Oreb, "Power spectral density analysis of optical substrates for gravitational-wave interferometry," Appl. Opt. 38, 4790-4801 (1999).

16. R. Blazey, "Light scattering by laser mirrors," Appl. Opt. 6, 831-836 (1967)

17. M. L. Zanaveskin, B. S. Roshchin, Y. V. Grishchenko, V. V. Azarova, V. E. Asadchikov, and A. L. Tolstikhina, "Correlation between the substrate roughness and light loss for interference mirror coatings," Cryst. Rep. 53, 701-707 (2008).

18. Y. L. Grand, J. P. Tach, and A. L. Floch, "Sensitive diffractionloss measurements of transverse modes of optical cavities by the decay-time method," J. Opt. Soc. Am. B 7, 1251-1253 (1990)

19. G. M. Harry, T. P. Bodiya, and R. De Salvo, eds., Optical Coatings and Thermal Noise in Precision Measurement (Cambridge University, 2012).

20. J. C. Stover, Optical Scattering 2nd ed. (SPIE, 1995).

21. C. Bruegge, N. Chrien, and D. Haner, "A spectralon brf data base for misr calibration applications," Remote Sens. Environ. 77, 354-366 (2001).

22. A. Bhandari, B. Hamre, Ø. Frette, L. Zhao, J. J. Stamnes, and M. Kildemo, "Bidirectional reflectance distribution function of spectralon white reflectance standard illuminated by incoherent unpolarized and plane-polarized light," Appl. Opt. 50, 2431-2442 (2011)

23. L. J. S. I. Michael and R. Cohen, "Diffuse reflectance measurements of standard diffusers," http://www.4physics.com/tn3/ lambertian.htm.

24. P. Y. Bely, ed., The Design and Construction of Large Optical Telescopes (Springer-Verlag, 2003).

25. B. Kells, "Scattered light loss from LIGO arm cavity mirrors," LIGO DCC T0900128-v3 (2009). https.dcc.ligo.org/cgi-bin/ DocDB/ShowDocument?docid=1521 